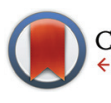

CrossMark \& click for updates

Cite this: Dalton Trans., 2015, 44 21099

Received 9th September 2015, Accepted 11th November 2015 DOI: $10.1039 / \mathrm{c} 5 \mathrm{dt} 03518 \mathrm{j}$ www.rsc.org/dalton

\title{
The formation mechanism of iron oxide nanoparticles within the microwave-assisted solvothermal synthesis and its correlation with the structural and magnetic properties
}

\author{
Z. Kozakova,* I. Kuritka, N. E. Kazantseva, V. Babayan, M. Pastorek, M. Machovsky, \\ P. Bazant and P. Saha
}

\begin{abstract}
Magnetic nanoparticles based on $\mathrm{Fe}_{3} \mathrm{O}_{4}$ were prepared by a facile and rapid one-pot solvothermal synthesis using $\mathrm{FeCl}_{3} \cdot 6 \mathrm{H}_{2} \mathrm{O}$ as a source of iron ions, ethylene glycol as a solvent and $\mathrm{NH}_{4} \mathrm{Ac}_{1}\left(\mathrm{NH}_{4}\right)_{2} \mathrm{CO}_{3}$, $\mathrm{NH}_{4} \mathrm{HCO}_{3}$ or aqueous $\mathrm{NH}_{3}$ as precipitating and nucleating agents. In contrast to previous reports we reduce the synthesis time to 30 minutes using a pressurized microwave reactor without the requirement of further post-treatments such as calcination. Dramatically reduced synthesis time prevents particle growth via Ostwald ripening thus the obtained particles have dimensions in the range of 20 to $130 \mathrm{~nm}$, they are uniform in shape and exhibit magnetic properties with saturation magnetization ranging from 8 to $76 \mathrm{emu} \mathrm{g}^{-1}$. The suggested method allows simple particle size and crystallinity tuning resulting in improved magnetic properties by changing the synthesis parameters, i.e. temperature and nucleating agents. Moreover, efficiency of conversion of raw material into the product is almost $100 \%$.
\end{abstract}

\section{Introduction}

Recently, the interest in preparation of nano-sized magnetic particles has risen due to their potential utilization in many areas of use. The most ambitious applications are in medicine and pharmacy for magnetic resonance imaging, biomaterial diagnostics, and drug delivery and in cancer treatment: magnetically mediated hyperthermia. Furthermore, they are used in the preparation of functional magnetic materials, high recording media, coloured pigments and ferrofluids. ${ }^{1-6}$ All of these applications require defined nanoparticle features together with high magnetization. Therefore, the development of a synthetic method enabling the preparation of a tailormade product with a truly wide range of required properties attracts great attention. Lots of methods have been introduced for preparation of magnetite nanoparticles, by using both, dry and wet chemistry. Thanks to the simplicity of the solvothermal method, it became one of the most popular among them. It is based on the principle of heating a solution of a metallic salt in a suitable solvent in the Teflon-lined stainless autoclave in the presence of other substances, such as nucleating or reducing agents and surfactants. Recently, several groups have described a relatively simple preparation of well-crystallized

Centre of Polymer Systems, Tomas Bata University in Zlin, tr. Tomase Bati 5678, 76001 Zlin, Czech Republic. E-mail: zkozakova@cps.utb.cz magnetic nanoparticles where ferric chloride is the source of iron cations and ethylene glycol serves both as a solvent and a reducing agent. Moreover, other substances used in synthesis were ethylenediamine serving as both, a coordinating agent and a quasi-surfactant, urea or different ammonium salts used as nucleating agents, and sodium acetate or other chemicals. ${ }^{7-10}$ However, tardiness of all of these methods (synthesis time at least 12 hours) is their main disadvantage which should be solved in order to improve the efficiency and applicability of solvothermal syntheses. Microwave heating, using the transformation of electromagnetic energy to heat, seems to be a suitable alternative to conventional heating leading to acceleration of the synthetic procedure. Common microwave reactors use radiation of frequency $2.45 \mathrm{GHz}$ and wavelength $12.2 \mathrm{~cm}$. Microwave synthesis is used in both organic and inorganic chemistry as a rapid method durable usually for several minutes and therefore it is also energy saving. It is possible to operate with a reflux system at atmospheric pressure that provides preparation of particles of various sizes and shapes, such as spheres, sheets, rods, tubes and others as it was demonstrated recently. ${ }^{11-13}$ Several microwave-assisted methods utilizing the reflux system have been used for synthesis of magnetic particles. However, these methods are relatively tedious and time-consuming as it turned out. As an example, two hours are required for preparation of $\mathrm{Fe}_{3} \mathrm{O}_{4}$ nanoparticles by MW heating of the product obtained by mixing the solutions of $\mathrm{FeCl}_{3}$ and $\mathrm{FeSO}_{4}$ under argon protec- 
tion with the addition of aqueous $\mathrm{NH}_{3} .{ }^{14}$ In another case, magnetite and maghemite nanoparticles were synthesized by the fast and simple method based on MW heating of a precursor obtained from the mixture of $\mathrm{FeCl}_{3}$ and polyethylene glycol $\left(M_{\mathrm{w}}=20000\right)$ in distilled water with the addition of hydrazine hydrate; however, the as-obtained particles have relatively wide size distribution and irregular shapes. ${ }^{15}$ According to our experience, heating limited by boiling temperature, intense mass flows accompanying the evaporation and subsequent condensation of the solvent in external coolers and foaming of the reaction mixture are strong limitations for these systems. Although a pressurized system for solvothermal synthesis offers relatively simple control over the morphology and particle size, examples of microwave pressurized reactors are reported very rarely in this field. Caillot et al. used a microwave reactor connected with an autoclave (RAMO system, Reacteur Autoclave Micro Onde) for the synthesis of magnetic nanoparticles using $\mathrm{FeCl}_{2} \cdot 4 \mathrm{H}_{2} \mathrm{O}$ and sodium ethoxide as the starting material. ${ }^{16}$ The resulting products were $\mathrm{Fe}_{2} \mathrm{O}_{3}, \mathrm{Fe}_{3} \mathrm{O}_{4}$ or their mixtures depending on the concentration of sodium ethoxide. Microwave-assisted syntheses using a pressurized system are based on the exposure of reaction mixtures to MW radiation in sealed vessels made of PTFE (Teflon) or even glass in microwave ovens. Products of such synthesis are influenced by the initial setting of synthetic parameters, namely the concentration of metallic salts and the type of solvent. On the other hand, a programmable MW system with a precise control of temperature and pressure may offer full control over the synthesis conditions. ${ }^{17}$ In many cases, the temperature increase is needed for initiation of nucleation in nanoparticle synthesis. If conventional heating is used, the reaction mixture is heated through the wall of the reaction vessel via conduction and convection of heat, and thus the created temperature gradient causes non-uniform nucleation and growth conditions. Microwave heating can solve this problem, since the energy is directly transferred to the microwave absorbing materials and the total volume of the reactant is therefore heated, while the uniformity of heating is assured by the positioning of reaction vessels on a rotating carousel thus overcoming the main drawback of multimode MW ovens. ${ }^{18}$ There are two MW heating effects that determine the quality of the final substance: thermal and non-thermal effects. The thermal effect is considered as a fast and effective heating of the reaction mixture providing uniform nucleation and growth conditions and thus leads to preparation of uniform nanoparticles with small sizes and high crystallinity. Non-thermal effects include the creation of hot spots and hot surfaces during the heating of solid materials on the liquid-solid surfaces which support the reduction of metal precursors, nucleation and formation of metal clusters. ${ }^{11}$ These effects are still a matter of intensive discussion and controversies in synthetic chemistry. Moreover, the mechanisms of microwave effects playing the role in solvothermal synthesis of magnetic nanoparticles remain uncovered in the recent literature. The goal of this work is threefold. First is to establish a simple, fast and highly efficient method of magnetic nanoparticle synthesis with a view to the scientific and commercial uses. Towards this purpose we decided to combine the simplicity of solvothermal methods and the efficiency of microwave heating into a novel, environmentally friendly method providing the product of fine quality in high yields. Next, this work contributes to the elucidation of effects of microwave heating on the processes and reactions that occur during the synthesis. Understanding the correlation among the reaction mechanism, conditions and properties of resulting particles enables fulfilment of the third goal - development of a simple method with the possibility to tailor the properties of the product.

\section{Materials and methods}

\section{Solvothermal synthesis of magnetic nanoparticles}

Nano- and submicro-sized $\mathrm{Fe}_{3} \mathrm{O}_{4}$ particles were prepared by a simple solvothermal method in ethylene glycol solution with the help of microwave irradiation. All reagents used for the synthesis were purchased from Penta Ltd (Czech Republic) of analytical grade and used without further purification. In a standard experiment, $5 \mathrm{mmol}$ of $\mathrm{FeCl}_{3} \cdot 6 \mathrm{H}_{2} \mathrm{O}(1.352 \mathrm{~g})$ was dissolved in $60 \mathrm{~mL}$ of ethylene glycol, followed by the addition of $50 \mathrm{mmol}$ of ammonium acetate $-\mathrm{NH}_{4} \mathrm{Ac}(3.854 \mathrm{~g})$. This mixture was placed in a $100 \mathrm{~mL}$ Teflon reaction vessel (XP-1500 Plus), heated in a CEM Mars 5 microwave oven to the required temperature $\left(200{ }^{\circ} \mathrm{C}, 210{ }^{\circ} \mathrm{C}, 220{ }^{\circ} \mathrm{C}\right)$ and maintained at this temperature for 30 minutes. After the reaction, the vessel was cooled to room temperature and a black precipitate was collected with the help of a permanent magnet. Subsequently, the as-obtained product was washed with distilled water and ethanol for several times and dried naturally in air. Furthermore, in similar experiments, $25 \mathrm{mmol}$ of $\left(\mathrm{NH}_{4}\right)_{2} \mathrm{CO}_{3}(2.403 \mathrm{~g})$, $50 \mathrm{mmol}$ of $\mathrm{NH}_{4} \mathrm{HCO}_{3}(3.953 \mathrm{~g})$ or $50 \mathrm{mmol}$ of $\mathrm{NH}_{3}$ in the form of aqueous solution (3.8 $\mathrm{mL}$ of $25 \%$ water solution) was used as a nucleating agent instead of $\mathrm{NH}_{4}$ Ac. Further experiments involved the addition of 2 to $4 \mathrm{~mL}$ of demineralized water to the reaction system while the rest of parameters remained unchanged. In all of the experiments, $\mathrm{Fe}(\mathrm{III})$ was used as the only source of iron since there is no necessity to work under Ar (or other inert atmosphere) to prevent $\mathrm{Fe}$ (II) from oxidizing into $\mathrm{Fe}(\mathrm{III})$ and no need to work at a high $\mathrm{pH}$ to obtain uniform particles. Moreover, the reducing agent used, ethylene glycol, reduces $\mathrm{Fe}(\mathrm{III})$ to $\mathrm{Fe}(\mathrm{II})$ thus both oxidation states are present in the reaction system.

\section{Characterization methods}

First of all, the macroscopic appearance of the prepared powder was observed by naked eyes and by using a digital microscope DVM 2500 (Leica Microsystems, Germany). The structure of the final product was characterized by X-ray Diffraction (PANalytical X'Pert PRO) with $\mathrm{Cu} \mathrm{K} \alpha 1$ radiation $(\lambda=$ $1.540598 \AA$ ). Phase composition and crystallite sizes were determined according to Rietveld analysis. Particle size and morphology were preliminarily investigated with the help of Scanning Electron Microscopy (VEGA \|LMU, Tescan). The 
particle shape, organization and proportions were further specified by Transmission Electron Microscopy (JEOL 1200, JEOL). Image analysis was used for estimation of the particle size distribution. Magnetic properties were measured by a vibrating sample magnetometer (VSM 7400, Lake Shore).

\section{Results and discussion}

\section{Correlation of particle properties with reaction conditions}

The X-ray diffraction (XRD) patterns of samples exemplified on materials prepared with $\mathrm{NH}_{4} \mathrm{Ac}$ at 200,210 and $220{ }^{\circ} \mathrm{C}$ for 30 minutes can be seen in Fig. 1. The diffraction pattern of the material prepared at $220{ }^{\circ} \mathrm{C}$ is clearly attributed to the cubic crystal structure of $\mathrm{Fe}_{3} \mathrm{O}_{4}$, however, the presence of peaks that do not belong to the magnetite are observed in the patterns of materials prepared at 200 and $210{ }^{\circ} \mathrm{C}$, indicating creation of other crystalline phases at lower synthesis temperatures. In addition, although the diffraction pattern of the product of synthesis proceeding at $200{ }^{\circ} \mathrm{C}$ includes broadened regions mostly seen between 30 and $40^{\circ} 2 \theta$ that indicate the presence of amorphous portion and broad peaks characteristic of very small particles, these features gradually disappear while the synthesis temperature increases, and at $220^{\circ} \mathrm{C}$ are even absent. Therefore we can consider this system very sensitive within the range of a few tens of degrees centigrade and exploit the elevated temperature to accelerate the nucleation and growth of the nanoparticles of the required crystalline phase. The demonstrated development of sample crystallinity

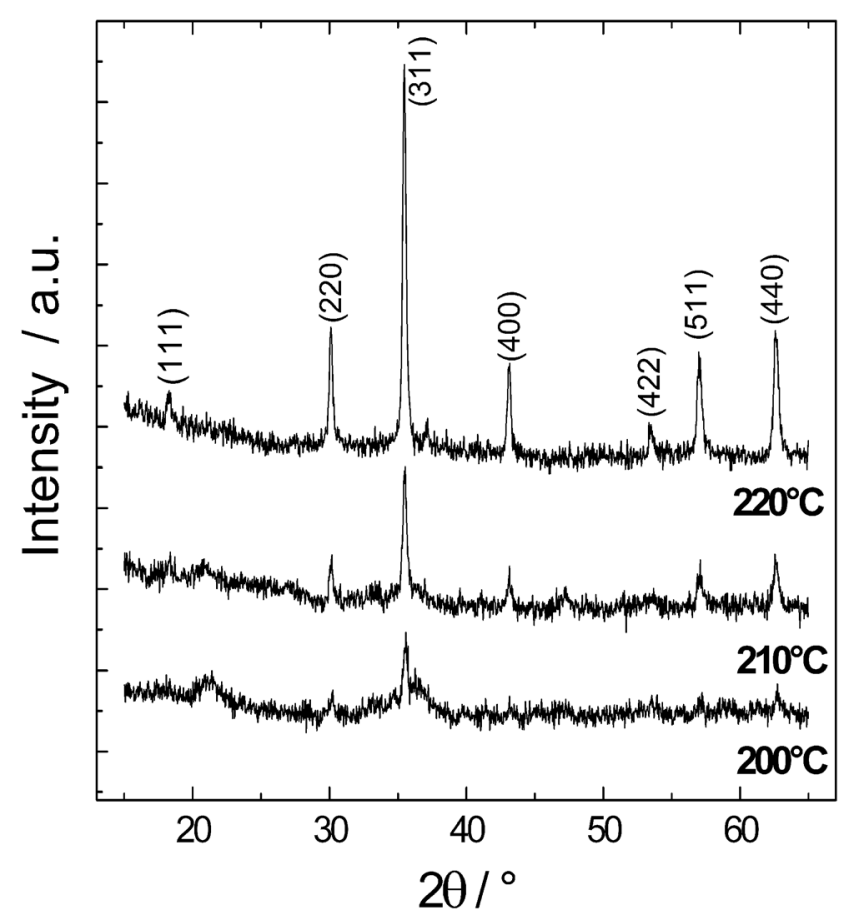

Fig. 1 XRD patterns of magnetic particles prepared with $\mathrm{NH}_{4} \mathrm{Ac}$ at various temperatures for 30 minutes. with the temperature increment is also followed by the set of the samples nucleated by other ammonium salts used in our experiments; however, it is not shown here for the sake of brevity. Calculation according to the Rietveld analysis showed that the crystallite size of the magnetite phase depends on the used nucleating agent and varies from 20 to $80 \mathrm{~nm}$. Phase composition also differs with the manner of nucleation. While the product prepared with aqueous ammonia is composed of pure $\mathrm{Fe}_{3} \mathrm{O}_{4}$ without other crystalline impurities, the presence of minor ferric compounds such as $\mathrm{FeO}(\mathrm{OH})$ and $\alpha-\mathrm{Fe}_{2} \mathrm{O}_{3}$ is evident in samples nucleated by ammonium carbonate and ammonium bicarbonate and utilization of ammonium acetate leads to the formation of the product with $\mathrm{FeO}(\mathrm{OH})$ in bigger portions.

It is well known that maghemite always accompanies magnetite in all materials due to the slow oxidation of magnetite into maghemite by air oxygen and results in the formation of the maghemite layer on the surface. In nanoscaled materials, this effect becomes considerable due to the large surface area and can be observed even in single crystals. ${ }^{19-21}$ Moreover, the reactants and the mechanism (will be discussed later) of the presented synthesis allows formation of maghemite and magnetite. Usually, the maghemite phase can be determined by Mössbauer spectrometry. ${ }^{22,23}$ However, the differentiation between the non-stoichiometric magnetite and magnetitemaghemite mixture is considerably challenging and was even claimed to be almost impossible. ${ }^{24,25}$ The same spinel structure and almost identical lattice parameters make identification of magnetite ( $\left.\mathrm{Fe}(\mathrm{II}) \mathrm{Fe}(\mathrm{III})_{2} \mathrm{O}_{4}\right)$ and maghemite $\left(\gamma\right.$ - $\left.\mathrm{Fe}(\mathrm{III})_{2} \mathrm{O}_{3}\right)$ by the $\mathrm{XRD}$ technique complicated. However, a deep detailed analysis

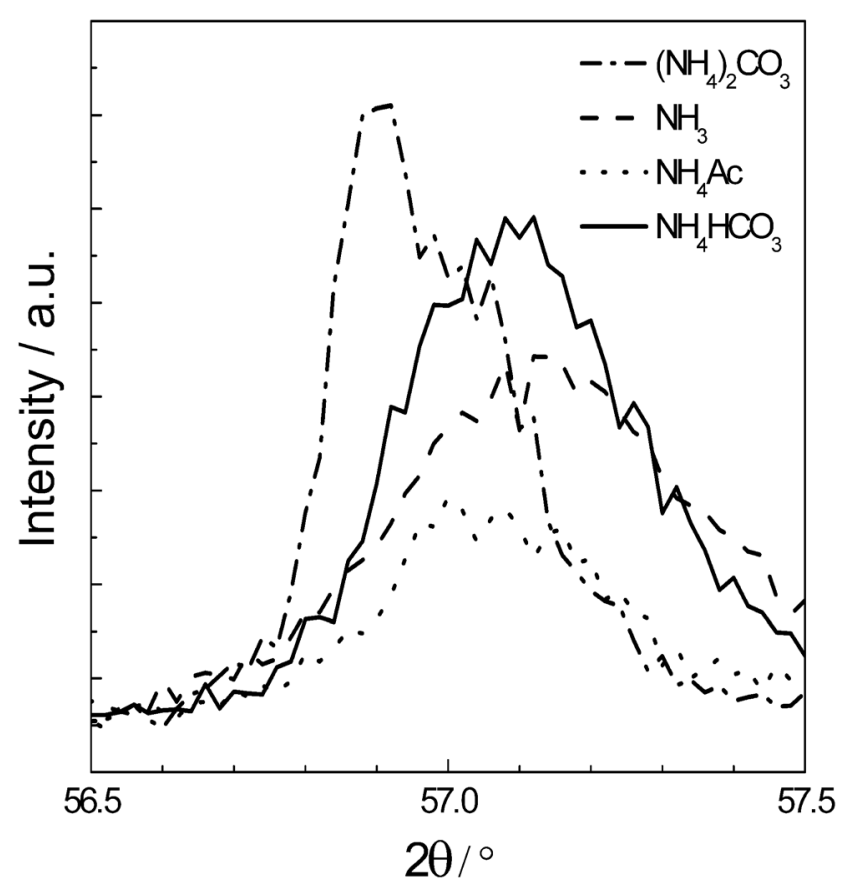

Fig. 2 Distinguishing between the magnetite and maghemite by detailed analysis of the diffraction peaks localized in the angle range $56.5-57.5^{\circ} 2 \theta \theta$. 


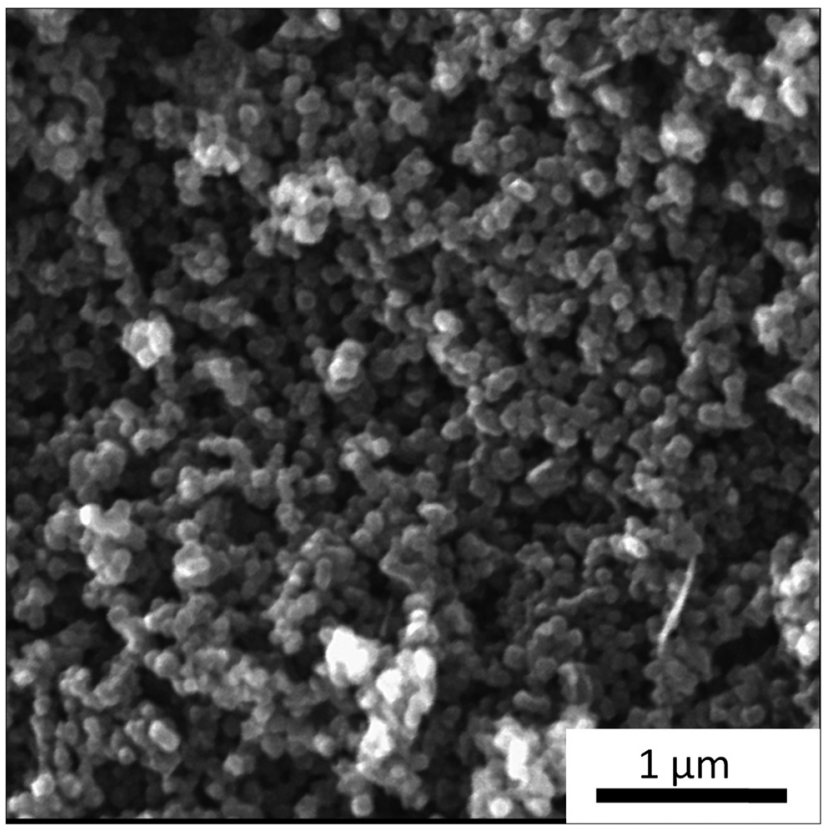

Fig. 3 SEM image of material prepared with $\mathrm{NH}_{4} \mathrm{Ac}$ for 30 minutes. of the (511) Bragg peak at the $2 \theta$ range $56.5-57.5^{\circ}$ and the (440) peak at the $2 \theta$ range $62-63.5^{\circ}$ provides insight into this issue. ${ }^{26}$ Pure magnetite has the central position of the diffraction peak at $57.0^{\circ}$ while maghemite has this peak slightly shifted to higher values, i.e. $57.3^{\circ}$. Under certain conditions, the observed peaks can be analysed by deconvolution with the use of suitable magnetite and maghemite standards. A detailed analysis of XRD patterns obtained for our samples are shown in Fig. 2. It is evident that all peaks are composed of more than one Gaussian contribution which can be attributed to the presence of both magnetite and maghemite phases in all samples. On the other hand, the peak positions at $X$-axes can be slightly shifted because the used diffractometer is not equipped with the Göbel mirror. Without the use of high resolution XRD and pure magnetite and maghemite nanoparticulate standards, the deconvolution cannot be successfully performed and the ratio between the phases cannot be exactly estimated. A scanning electron microscopy image (SEM) of particles prepared with ammonium acetate is shown in Fig. 3. The particles have a quasi-spherical shape and occur in the form of clusters. Similarly, an SEM study of all types of prepared products provides analogous results, which can be influenced by the resolution of the used method. The images of better resolution were obtained by transmission electron microscopy (TEM) (Fig. 4). Analyses of the
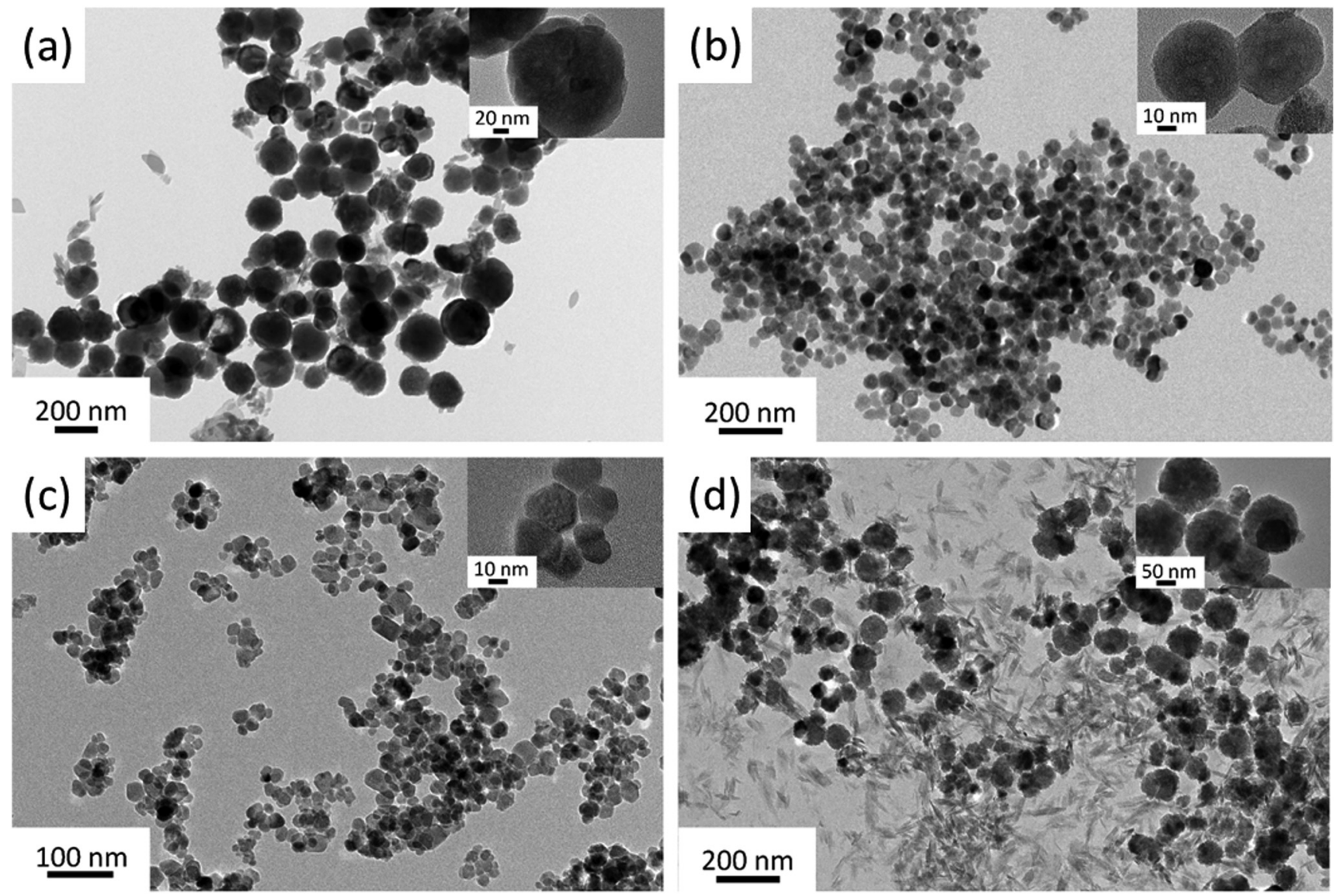

Fig. 4 TEM images of materials prepared at $220^{\circ} \mathrm{C}$ for 30 minutes with $\left(\mathrm{NH}_{4}\right)_{2} \mathrm{CO}_{3}$ (a), $\mathrm{NH}_{4} \mathrm{HCO}_{3}$ (b), aq. $\mathrm{NH}_{3}$ (c) and $\mathrm{NH}_{4} \mathrm{Ac}$ (d). 
TEM images show that the importance of a nucleation agent on the particle size, shape and organization is evident. The use of aqueous $\mathrm{NH}_{3}$ as well as $\mathrm{NH}_{4} \mathrm{HCO}_{3}$ provides a single-crystalline material composed of uniform-shaped polyhedral nanoparticles with narrow size distribution and dimension below $30 \mathrm{~nm}$. However, the use of $\left(\mathrm{NH}_{4}\right)_{2} \mathrm{CO}_{3}$ and $\mathrm{NH}_{4} \mathrm{Ac}$ for nucleation lead to the creation of clusters consisting of nano-sized grains and ranging to several hundred nanometers. The hierarchical structure of clusters affects the magnetic properties of materials obtained and therefore we further studied the reason for their formation within the microwave-assisted solvothermal process. The size of clusters obtained with the use of ammonium acetate based on TEM analysis is $60 \mathrm{~nm}$. Since the size of grains of the magnetite phase calculated by Rietveld analysis is about $40 \mathrm{~nm}$, these clusters seem to be formed by the binding of the small crystallites of the non-magnetic phase together with the magnetite crystals, as can be seen in the TEM image at high magnification (Fig. 4d). While the ammonium carbonate is used, particles with a diameter of about $120 \mathrm{~nm}$ are formed due to the twinning of crystallites of magnetite with a diameter of $80 \mathrm{~nm}$ induced by the incorporation of crystalline impurities (such as $\mathrm{FeO}(\mathrm{OH})$ and $\alpha-\mathrm{Fe}_{2} \mathrm{O}_{3}$ ) produced during the synthesis. Nanoparticle sizes are confirmed by calculations based on Rietveld analysis as described above.

Information on particle sizes obtained by TEM image analyses was further used for creation of histograms which give us a conception of particle size distribution in the prepared materials (Fig. 5).

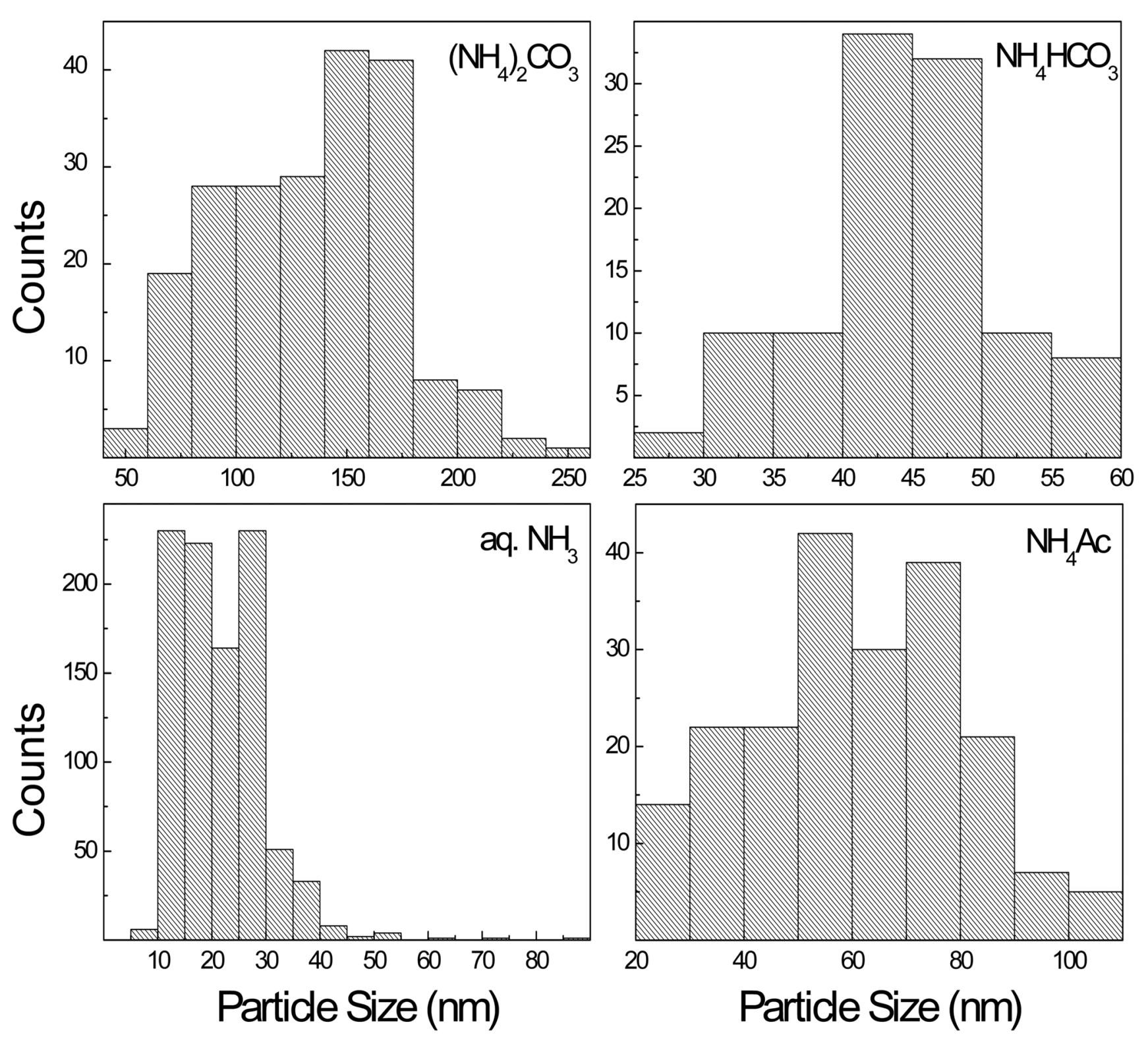

Fig. 5 Size distribution histograms of materials prepared at $220^{\circ} \mathrm{C}$ for 30 minutes with various nucleating agents. 
The magnetostatic properties of samples were investigated by using a vibrating sample magnetometer (VSM) at room temperature. Fig. 6 (left) demonstrates the magnetization curves of the material prepared with ammonium acetate synthesized at 200, 210 and $220^{\circ} \mathrm{C}$. Saturation magnetization $\left(M_{\mathrm{s}}\right)$ of nanoparticles obtained with $\mathrm{NH}_{4} \mathrm{Ac}$ varies from 13.8 to $67.7 \mathrm{emu} \mathrm{g}^{-1}$ with regard to the increase of temperature. The increase of $M_{\mathrm{S}}$ can be explained on the one hand to the crystallinity improvement seen in Fig. 1, and on the other hand probably due to the phase transition at higher temperature which led to formation of the material with a major magnetite (maghemite) fraction. Similar results were obtained for the samples nucleated by $\left(\mathrm{NH}_{4}\right)_{2} \mathrm{CO}_{3}$ with saturation magnetization ranging from 8.4 to $46.2 \mathrm{emu} \mathrm{g}^{-1}$, as well as for $\mathrm{NH}_{4} \mathrm{HCO}_{3}$ with $M_{\mathrm{s}}$ in the range of 33.2 to $75.2 \mathrm{emu}^{-1}$ and, also for aqueous $\mathrm{NH}_{3}$ with the highest saturation magnetization among the forenamed materials that reaches 44.5 to $76.3 \mathrm{emu}^{-1}$. Lower values of $M_{\mathrm{s}}$ of the prepared materials than that of the bulk magnetite (92-100 emu $\left.\mathrm{g}^{-1}\right)^{27}$ are common for nanoparticulate systems and can be attributed to the canted spins on the surfaces. ${ }^{28}$ On the other hand, the maghemite phase present on the surface of nanoparticles could also lead to the decrease of $M_{\mathrm{s}}$. For a better understanding of effects influencing the magnetic properties of the prepared materials, the magnetization curves obtained with the use of different nucleating agents were plotted together in Fig. 6 (right). Obviously, particles prepared with aqueous ammonia and ammonium bicarbonate have the highest saturation magnetization; particles prepared with ammonium carbonate have the lowest one. It is well known that coercivity is determined by effective magnetic anisotropy and thus depends on the material composition as well as the grain size and shape. Fig. 6 shows the rise of the coercivity depending on the synthesis temperature. It can be attributed to the already mentioned presence of the magnetite phase in the sample at the expense of other less magnetic phases such as hematite or non-magnetic goethite and the growth of the particles to the larger dimensions. However, despite variations of the particle size with the use of different nucleating agents, the coercivity of the materials prepared by different ways of nucleation is very similar and reaches about 60 Oe. Nevertheless, the material prepared with the use of ammonium carbonate is the only exception with nearly zero value of coercivity. The squeezed shape of the hysteresis loop (see the inset graph in Fig. 6) indicates the pronounced influence of the cluster formation on the hysteresis and confirms the presence of two magnetic phases that differ in magnetic properties. ${ }^{29}$

\section{The mechanism of nanoparticle formation by MW-assisted synthesis}

As the starting point for the description of the growth of $\mathrm{Fe}_{3} \mathrm{O}_{4}$ nanoparticles, the two-stage growth model of nanoparticles in supersaturated solutions can be adopted. ${ }^{30}$ In the first stage, nucleation of primary nanocrystals occurs followed by their aggregation into the secondary nanoparticles and, especially, this second step has to be reconsidered for specific microwave conditions. We used $\mathrm{NH}_{4} \mathrm{Ac},\left(\mathrm{NH}_{4}\right)_{2} \mathrm{CO}_{3}, \mathrm{NH}_{4} \mathrm{HCO}_{3}$ and aqueous $\mathrm{NH}_{3}$ as nucleating agents. $\mathrm{NH}_{4} \mathrm{Ac}$ is a weak-acidweak-base salt that can be hydrolysed at high temperature in
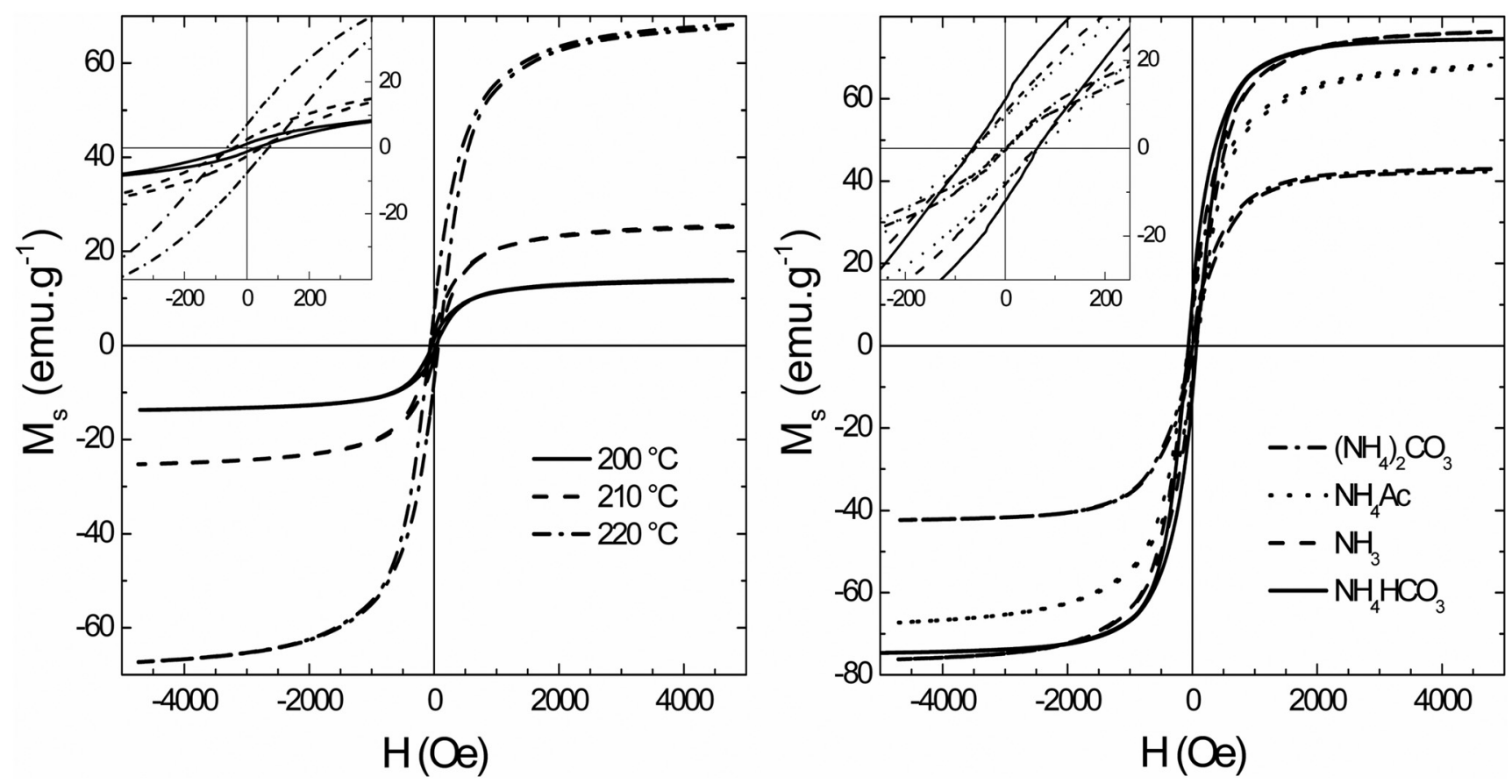

Fig. 6 Magnetization curves of materials prepared with $\mathrm{NH}_{4} \mathrm{Ac}$ at various temperatures for 30 minutes (left) and at $220{ }^{\circ} \mathrm{C}$ for 30 minutes with various nucleating agents (right). 
the presence of a trace amount of water coming from $\mathrm{FeCl}_{3} \cdot 6 \mathrm{H}_{2} \mathrm{O}$ as follows: ${ }^{7}$

$$
\mathrm{NH}_{4} \mathrm{Ac}+\mathrm{H}_{2} \mathrm{O} \rightleftharpoons \mathrm{HAc}+\mathrm{NH}_{3} \cdot \mathrm{H}_{2} \mathrm{O}
$$

Although $\mathrm{NH}_{3} \cdot \mathrm{H}_{2} \mathrm{O}$ is used in ref. 7, we do not expect the formation of solid ammonium hydrates in the system and we consider this formula to be an expression of the presence of ammonia and water $\left(\mathrm{NH}_{3}+\mathrm{H}_{2} \mathrm{O}\right)$ as it was most likely the author's original intention.

Similarly, $\left(\mathrm{NH}_{4}\right)_{2} \mathrm{CO}_{3}$ and $\mathrm{NH}_{4} \mathrm{HCO}_{3}$ can be hydrolysed into $\mathrm{NH}_{3}$ :

$$
\begin{gathered}
\left(\mathrm{NH}_{4}\right)_{2} \mathrm{CO}_{3}+\mathrm{H}_{2} \mathrm{O} \rightleftharpoons 2 \mathrm{NH}_{3}+2 \mathrm{H}_{2} \mathrm{O}+\mathrm{CO}_{2} \\
\mathrm{NH}_{4} \mathrm{HCO}_{3}+\mathrm{H}_{2} \mathrm{O} \rightleftharpoons \mathrm{NH}_{3}+2 \mathrm{H}_{2} \mathrm{O}+\mathrm{CO}_{2}
\end{gathered}
$$

$\mathrm{NH}_{3}$, either as the product of the above reactions or added as aqueous solution yields hydroxide anions.

$$
\mathrm{NH}_{3}+\mathrm{H}_{2} \mathrm{O} \rightleftharpoons \mathrm{NH}_{4}^{+}+\mathrm{OH}^{-}
$$

In this case, $\mathrm{Fe}(\mathrm{III})$ salt was used as the precursor. Precipitation of its hydroxide in the form of colloidal solution proceeds as follows:

$$
\mathrm{Fe}^{3+}+3 \mathrm{OH}^{-} \rightleftharpoons \mathrm{Fe}(\mathrm{OH})_{3}
$$

Next, formation of $\mathrm{Fe}_{2} \mathrm{O}_{3}$ particles may continue according to:

$$
2 \mathrm{Fe}(\mathrm{OH})_{3} \rightleftharpoons \mathrm{Fe}_{2} \mathrm{O}_{3}+3 \mathrm{H}_{2} \mathrm{O}
$$

Hence the maghemite phase can be formed in this way.

Under the presence of a mild reducing agent (e.g. ethylene glycol), Fe(II) may appear in the system. Ethylene glycol can undergo dehydration and the so-formed acetaldehyde $\mathrm{e}^{31,32}$ reduces $\mathrm{Fe}(\mathrm{III})$ to $\mathrm{Fe}$ (II) and gives ferrous hydroxide in the form of a green colloid:

$$
\begin{gathered}
2 \mathrm{HOCH}_{2}-\mathrm{CH}_{2} \mathrm{OH} \rightleftharpoons 2 \mathrm{CH}_{3} \mathrm{CHO}+2 \mathrm{H}_{2} \mathrm{O} \\
2 \mathrm{CH}_{3} \mathrm{CHO}+2 \mathrm{Fe}^{3+} \rightleftharpoons \mathrm{CH}_{3} \mathrm{CO}-\mathrm{COCH}_{3}+2 \mathrm{Fe}^{2+}+2 \mathrm{H}^{+} \\
\mathrm{Fe}^{2+}+2 \mathrm{OH}^{-} \rightleftharpoons \mathrm{Fe}(\mathrm{OH})_{2}
\end{gathered}
$$

Most likely, both ferric and ferrous oxidation states of iron coexist in the reaction mixture thus enabling magnetite formation:

$$
2 \mathrm{Fe}(\mathrm{OH})_{3}+\mathrm{Fe}(\mathrm{OH})_{2} \rightleftharpoons \mathrm{Fe}_{3} \mathrm{O}_{4}+4 \mathrm{H}_{2} \mathrm{O}
$$

A minor phase, goethite, was observed and its presence in the prepared materials can be explained by partial dehydration:

$$
\mathrm{Fe}(\mathrm{OH})_{3} \rightleftharpoons \mathrm{FeO}(\mathrm{OH})+\mathrm{H}_{2} \mathrm{O}
$$

or by the following equation in the presence of oxygen:

$$
4 \mathrm{Fe}(\mathrm{OH})_{2}+\mathrm{O}_{2} \rightleftharpoons 4 \mathrm{FeO}(\mathrm{OH})+2 \mathrm{H}_{2} \mathrm{O}
$$

The first step of nucleation starts with the first appearance of heterogeneity in the system similarly, as in common solvothermal methods. Hu et al. ${ }^{7}$ proposed the mechanism with the key role of $\mathrm{NH}_{3} \cdot \mathrm{H}_{2} \mathrm{O}$. Aqueous ammonia evaporates and forms gaseous bubbles that provide heterogeneous nucleation centers for newly-formed nanoparticles, which can then aggregate around the gas-liquid interface. In this case, we used various precipitation agents and all of them lead to nanocrystalline products in the same reaction time; however, we did not obtain any evidence of hollow structures. Moreover, other authors used ammonia-less techniques with sodium acetate and obtained similar results, albeit this was achieved without MW assistance. ${ }^{30,33}$ Lou et al. in their review ${ }^{34}$ consider a gas $^{3}$ templating mechanism that uses soluble gases especially that originated from the decomposition of organic molecules such as urea, as highly speculative. Therefore we propose that the reaction starts with the formation of colloid according to the eqn (5) and (9) rather than with the formation of nanobubbles.

In the second step, it is generally accepted for conventional solvothermal methods that the freshly formed nanocrystals are unstable due to high surface energy so they tend to aggregate. The driving force for this aggregation is the pursuit of reducing the surface energy by both attachment among the primary nanocrystals and their rotation caused by various interactions including the Brownian motion or short-range interactions. $^{30,35}$ In our case of MW heating, the solvent at high temperature (above $200^{\circ} \mathrm{C}$ ) becomes less absorbing due to the decrease of dielectric constant and the loss factor thus the solvent becomes virtually more transparent for microwaves, which means that the ionic mechanism of microwave absorption may be prevailing over the dipoles. ${ }^{12}$ After the solid particles occur in the solution, another mechanism becomes active due to particle surface polarization that might selectively influence their growth. Moreover, as the heat is generated at the surface of nanoparticles, the local temperature gradient can contribute to the hydroxide-to-oxide transformation described in eqn (6) and (10). Once the oxide particle reaches the critical size corresponding to the single-domain state, another mechanism of MW absorption is assumed to take the main role. Microwave energy is transformed into heat by magnetic moment rotation and after some time in bigger multidomain particles also by the magnetic domain wall motion. This sequence of several mechanisms can explain the sudden formation of particles in the reaction mixture as well as the very short synthesis time. The time required for the formation of nanoparticles with magnetic properties exceeds 12 hours for common solvothermal synthesis. Assuming simply just the fast heating of the reaction mixture and no non-thermal effects supporting the transformation of the raw material into the product we reduced the synthesis time firstly to 20 minutes. However, the time of microwave treatment was not sufficient for the precipitate formation and only opaque yellow colloid was obtained with no magnetically separable fraction. Prolongation of the exposure to microwaves to 30 minutes enables formation of a black precipitate and, moreover, the conversion of the starting material into the product runs with almost $100 \%$ efficiency. This fact refers to the sudden formation of the final product, and the relatively narrow particle size distribution and supports the concept of 
sequential action of MW absorption mechanisms as a nonthermal effect.

The role of solvent has to be reconsidered in comparison with the conventional processes as well. Ethylene glycol is an exceptionally good microwave absorbing solvent at $2.45 \mathrm{GHz}$ $(\tan \delta=1.350)^{36}$ which assures very fast increase of temperature at the beginning of the synthesis together with fast achievement of quasi-stable reaction conditions in the pressurized reaction vessel. On the other hand, it is not directly involved in the chemistry of ferric hydroxide or oxide formation. EG can disproportionate according to eqn (7) but the equilibrium must be shifted to the left side of the reaction, however the acetaldehyde enters the redox reaction (8) and yields $\mathrm{Fe}^{2+}$ cations. Liberation of one $\mathrm{H}_{2} \mathrm{O}$ molecule per each $\mathrm{Fe}^{2+}$ cation occurs according to eqn (7) and (8). A stoichiometric amount of water is delivered to the reaction system with $\mathrm{FeCl}_{3} \cdot 6 \mathrm{H}_{2} \mathrm{O}$. Water plays a crucial role in cation solvation, nucleation agent hydrolysis, see eqn (1)-(4), and in the formation of ferric and ferrous hydroxides, eqn (5) and (9). Hence, water is not present in excess but in stoichiometric amount as one of the reactants. Moreover, it has the highest dielectric constant among common solvents although its loss factor $(\tan \delta=0.123)^{36}$ is approximately eleven times lower than that of EG.

In contrast to fast nucleation in aqueous solutions, aggregation in ethylene glycol is kinetically slower due to the fewer hydroxyl groups on the particle surface and higher viscosity, which allows adequate rotation of nanocrystals to form a lowenergy configuration of interface and perfectly organized assemblies. Subsequently, these aggregates further crystallize and form compact crystals that exhibit features of singlecrystal particles. ${ }^{9}$ As it is possible to see in Fig. 3, the particles obtained with the use of aqueous $\mathrm{NH}_{3}$ are single crystals with a polyhedral shape, the second most perfectly developed crystallites were observed for $\mathrm{NH}_{4} \mathrm{HCO}_{3}$ then followed by less developed spherical multi-grains obtained by the use of $\left(\mathrm{NH}_{4}\right)_{2} \mathrm{CO}_{3}$, whereas nucleation by $\mathrm{NH}_{4} \mathrm{Ac}$ led to the biggest polycrystalline particles with a spherical shape. The same trend was observed for phase composition of the prepared materials. As can be seen in Table 1, the use of aqueous ammonia yielded a single phase material while $\mathrm{NH}_{4} \mathrm{Ac}$ gave the highest fraction of goethite in the product. The other nucleation agents $\mathrm{NH}_{4} \mathrm{HCO}_{3}$ and $\left(\mathrm{NH}_{4}\right)_{2} \mathrm{CO}_{3}$ gave moderate results. The observed trend of perfection of crystalline structure development correlates well with the virtually hidden presence of water in nucle- ating agents. Hydrolysis of $\mathrm{NH}_{4} \mathrm{Ac}$ does not provide any extra water molecule per each $\mathrm{NH}_{3}$ in eqn (1). Hydrolysis of $\left(\mathrm{NH}_{4}\right)_{2} \mathrm{CO}_{3}$ yields one extra $\mathrm{H}_{2} \mathrm{O}$ molecule per two $\mathrm{NH}_{3}$ and hydrolysis of $\mathrm{NH}_{4} \mathrm{HCO}_{3}$ yields two extra water molecules per each $\mathrm{NH}_{3}$, see eqn (2) and (3). About $2.5 \mathrm{~mL}$ of water gets to the reaction system with the addition of aqueous ammonia (25\% aqueous solution), which means approximately 3 molecules of $\mathrm{H}_{2} \mathrm{O}$ per each $\mathrm{NH}_{3}$. Since the synthesis conditions such as temperature and duration are the same in all four cases, the influence of composition of the reaction mixture on the particle shape and organization was further investigated. A similar system was described by Cao et al. ${ }^{35}$ and denominated as the (EG) $-\mathrm{H}_{2} \mathrm{O}$ system. By the addition of different amounts of water (1-3 mL) into the reaction system, $\mathrm{Fe}_{3} \mathrm{O}_{4}$ polyhedral particles with different sizes are formed whereas the samples prepared without water addition led to the formation of the product that consisted of spherical particles assembled by smaller particles. If water is added to the reaction system, the coordinated EG molecules will be substituted by water molecules since the coordination of water molecules to metal ions is stronger than that of EG molecules. By selecting the amount of water to be added, the particle size can be influenced in agreement with Cao et al. We considered the role of addition of deionized water as one of the most important factors in the control of particle size and size distribution, too. For this purpose we also performed experiments with the addition of water into the reaction mixture. According to the TEM images shown above, the sample nucleated by $\mathrm{NH}_{4} \mathrm{HCO}_{3}$ was chosen due to the narrow size distribution and shape uniformity of particles. Apparently from the TEM image seen in Fig. 7, addition of small amount of demineralized water causes reduction of particle size from 30 to $10 \mathrm{~nm}$, even if the crystalline composition and design of the as-prepared particles remain unchanged and they also possess a single-crystalline character with a polyhedral shape. The size of particles dropped to the value under which the particles are no longer in a single-domain state and we obtained superparamagnetic particles. This magnetic behaviour is desired for many applications, however, in many cases and also in this case, this transition also led to the decrease of $M_{\mathrm{s}}$ value (Fig. 7 (right)). Next to the synthetic conditions that can be directly controlled such as the synthetic temperature and composition of the reaction mixture, pressure in reaction vessels can be influenced only indirectly, through setting the level of the vessel filling, the type of solvent and temperature (reaction temperature below

Table 1 Variations of properties of materials prepared with different nucleating agents

\begin{tabular}{|c|c|c|c|c|c|c|}
\hline $\begin{array}{l}\text { Type of } \\
\text { nucleating agent }\end{array}$ & $\begin{array}{l}\mathrm{NH}_{3} \text { to } \mathrm{H}_{2} \mathrm{O} \\
\text { molecules ratio }\end{array}$ & $\begin{array}{l}\text { Crystalline } \\
\text { impurities }\end{array}$ & $\begin{array}{l}\mathrm{Fe}_{3} \mathrm{O}_{4} \text { crystallite } \\
\text { size by XRD (nm) }\end{array}$ & $\begin{array}{l}\text { Particle size } \\
\text { by TEM (nm) }\end{array}$ & $\begin{array}{l}M_{\mathrm{s}} \\
\left(\mathrm{emu} \mathrm{g}^{-1}\right)\end{array}$ & $H_{\mathrm{c}}(\mathrm{Oe})$ \\
\hline$\left(\mathrm{NH}_{4}\right)_{2} \mathrm{CO}_{3}$ & $1: 0.5$ & Minor $\mathrm{Fe}_{2} \mathrm{O}_{3} \cdot \mathrm{H}_{2} \mathrm{O}, \mathrm{Fe}_{2} \mathrm{O}_{3}$ & 84 & 130 & 46 & 2 \\
\hline $\mathrm{NH}_{4} \mathrm{HCO}_{3}$ & $1: 1$ & Minor $\mathrm{Fe}_{2} \mathrm{O}_{3} \cdot \mathrm{H}_{2} \mathrm{O}$ & 32 & 40 & 75 & 65 \\
\hline $\mathrm{NH}_{3}$ aq. & $1: 3$ & - & 17 & 20 & 76 & 61 \\
\hline $\mathrm{NH}_{4} \mathrm{Ac}$ & $1: 0$ & Minor $\alpha-\mathrm{FeO}(\mathrm{OH})$ & 42 & 60 & 68 & 67 \\
\hline
\end{tabular}



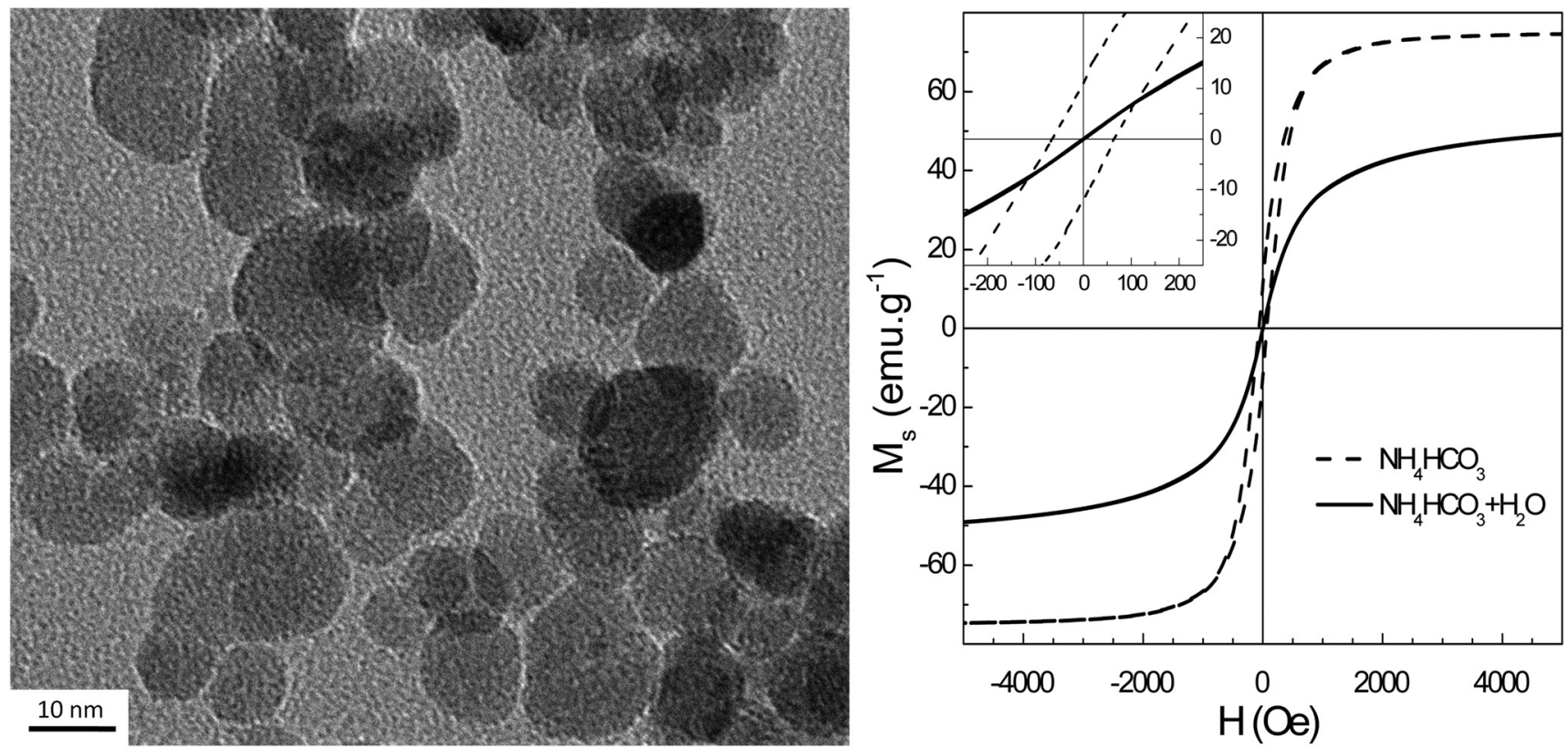

Fig. 7 TEM image of material prepared with $\mathrm{NH}_{4} \mathrm{HCO}_{3}$ with the addition of water (left) and magnetization curves of the same material with and without the water addition (right).

or above the boiling point of solvent) and also by the type of used reactant. Since the used solvent and the level of vessel filling was almost the same in our experiments, the pressure in reaction vessels during the synthesis depended on the used nucleation agents and varied from 900 to $3800 \mathrm{kPa}$. When aqueous ammonia and ammonium acetate were used within the synthesis, the lowest pressure $(800-1000 \mathrm{kPa})$ was obtained. On the other hand, the decomposition of ammonium carbonate was accompanied with increasing the pressure up to $3800 \mathrm{kPa}$ due to the significant change of mole ratio of the reaction (see eqn (2)). The use of ammonium bicarbonate leads to a medium value of pressure about $2000 \mathrm{kPa}$, since a smaller change of mole ratio of reaction accompanied the decomposition reaction.

The importance of the use of a pressurized reactor lies in the possibility to superheat the solvent by which the synthesis can be performed above the boiling point. Moreover, above the boiling point, ethylene glycol becomes virtually transparent for microwaves thus the reaction mixture behaves similarly as ionic liquids that consisted entirely of ions and the ionic effect becomes the prevailing reaction mechanism. The ionic effect is considered to be much stronger than the dipolar rotation mechanism and therefore the conversion of the raw material into the solid product in the reaction system can be significantly enhanced. Moreover, the use of pressurized microwave reactors with sealed vessels ensure a limited amount of oxygen from the atmosphere to be involved in the reaction system thus preventing the complete oxidation of $\mathrm{Fe}(\mathrm{II})$ into the $\mathrm{Fe}$ (III) cations that lead to the formation of undesired by-products such as $\alpha-\mathrm{Fe}_{2} \mathrm{O}_{3}$.

\section{Conclusions}

A simple and effective method for preparation of magnetic material based on $\mathrm{Fe}_{3} \mathrm{O}_{4}$ in nano- and submicron-dimension has been proposed. Efficiency of this method is to provide the transformation of a common solvothermal method using conventional heating into a microwave-assisted method. The change of heating method causes considerable acceleration of the reaction due to the direct heating of the absorbing material and non-thermal effects supporting the transformation of raw material into the final product. Thanks to the use of a pressurized system enabling the control of the synthesis temperature and time as well as indirectly total pressure in the reaction vessel, materials with various properties that reflect the requirements of the specific applications were prepared. Particles ranging from 20 to more than $100 \mathrm{~nm}$ based on singlecrystals or crystalline assemblies were prepared by selecting the type of nucleating agent.

It is established that an appropriate composition of the starting reaction mixture leads to the formation of the pure one-phase or multiphase materials. Magnetic properties of materials obtained by the proposed microwave-assisted method can be tailored as a consequence of the changes in the phase composition, crystalline structure, particles size, shape and their self-organization into clusters. Besides the change of reaction mixture by the variation of nucleating agents, the reaction medium represented by ethylene glycol can be modified by the addition of demineralized water. A small portion of demineralized water in the reaction medium results in the reduction of nanoparticle sizes to one-third of 
the original size that is significantly reflected in the magnetic properties and can lead to the formation of nanoparticles in superparamagnetic and ferrimagnetic states. Even the addition of minor amounts of demineralized water to the reaction system can serve as the tool for tailoring the magnetic properties of the final product and makes this microwave-assisted solvothermal method more flexible to the current requirements. Consequently, simplicity, low cost and variability and reproducibility of this method make it a suitable candidate for the routine preparation of ferrimagnetic and superparamagnetic materials in nano-dimensions.

\section{Acknowledgements}

This work was supported by the Ministry of Education, Youth and Sports of the Czech Republic - Program NPU I (LO1504).

\section{Notes and references}

1 A. K. Gupta and M. Gupta, Biomaterials, 2005, 26, 3995-4021.

2 D. K. Kim, Y. Zhang, J. Kehr, T. Klason, B. Bjelke and M. Muhammed, J. Magn. Magn. Mater., 2001, 225, 256-261.

3 A. S. Lubbe, C. Alexiou and C. Bergemann, J. Surg. Res., 2001, 95, 200-206.

4 S. W. Cao, Y. J. Zhu, M. Y. Ma, L. Li and L. Zhang, J. Phys. Chem. C, 2008, 112, 1851-1856.

5 J. Jestin, F. Cousin, I. Dubois, C. Menager, R. Schweins, J. Oberdisse and F. Boue, Adv. Mater., 2008, 20, 2533-2540.

6 C. Scherer and A. M. F. Neto, Braz. J. Phys., 2005, 35, 718-727.

7 P. Hu, L. J. Yu, A. H. Zuo, C. Y. Guo and F. L. Yuan, J. Phys. Chem. C, 2009, 113, 900-906.

8 X. Y. Chen, Z. J. Zhang, X. X. Li and C. W. Shi, Chem. Phys. Lett., 2006, 422, 294-298.

9 L. P. Zhu, H. M. Xiao, W. D. Zhang, G. Yang and S. Y. Fu, Cryst. Growth Des., 2008, 8, 957-963.

10 H. Deng, X. L. Li, Q. Peng, X. Wang, J. P. Chen and Y. D. Li, Angew. Chem., Int. Ed., 2005, 44, 2782-2785.

11 M. Tsuji, M. Hashimoto, Y. Nishizawa, M. Kubokawa and T. Tsuji, Chem. - Eur. J., 2005, 11, 440-452.

12 A. Loupy, Microwaves in organic synthesis - a review, Wiley VHC, Welhein, 2002.

13 P. Lidstrom, J. Tierney, B. Wathey and J. Westman, Tetrahedron, 2001, 57, 9225-9283.

14 R. Y. Hong, T. T. Pan and H. Z. Li, J. Magn. Magn. Mater., 2006, 303, 60-68.
15 W. W. Wang, Y. J. Zhu and M. L. Ruan, J. Nanopart. Res., 2007, 9, 419-426.

16 T. Caillot, D. Aymes, D. Stuerga, N. Viart and G. Pourroy, J. Mater. Sci., 2002, 37, 5153-5158.

17 Z. Kozakova, P. Bazant, M. Machovsky, V. Babayan and I. Kuritka, Acta Phys. Pol., A, 2010, 118, 948-949.

18 J. A. Gerbec, D. Magana, A. Washington and G. F. Strouse, J. Am. Chem. Soc., 2005, 127, 15791-15800.

19 A. P. Grosvenor, B. A. Kobe and N. S. McIntyre, Surf. Sci., 2004, 565, 151-162.

20 P. Guardia, A. Labarta and X. Batlle, J. Phys. Chem. C, 2011, 115, 390-396.

21 J. Tang, M. Myers, K. A. Bosnick and L. E. Brus, J. Phys. Chem. B, 2003, 107, 7501-7506.

22 A. G. Roca, J. F. Marco, M. D. Morales and C. J. Serna, J. Phys. Chem. C, 2007, 111, 18577-18584.

23 A. C. Doriguetto, N. G. Fernandes, A. I. C. Persiano, E. Nunes, J. M. Greneche and J. D. Fabris, Phys. Chem. Miner., 2003, 30, 249-255.

24 R. E. Vandenberghe, C. A. Barrero, G. M. da Costa, E. Van San and E. De Grave, Hyperfine Interact., 2000, 126, 247-259.

25 C. A. Gorski and M. M. Scherer, Environ. Sci. Technol., 2009, 43, 3675-3680.

26 W. Kim, C. Y. Suh, S. W. Cho, K. M. Roh, H. Kwon, K. Song and I. J. Shon, Talanta, 2012, 94, 348-352.

27 R. M. Cornell and U. Schwertmann, The iron oxides: structure, properties, reactions occurences, and uses, Wiley-VCH, Weinheim, 2003.

28 M. P. Morales, S. Veintemillas-Verdaguer, M. I. Montero, C. J. Serna, A. Roig, L. Casas, B. Martinez and F. Sandiumenge, Chem. Mater., 1999, 11, 3058-3064.

29 R. Skomski, J. Phys.: Condens. Matter, 2003, 15, R841-R896.

30 S. H. Xuan, Y. X. J. Wang, J. C. Yu and K. C. F. Leung, Chem. Mater., 2009, 21, 5079-5087.

31 S. E. Skrabalak, B. J. Wiley, M. Kim, E. V. Formo and Y. N. Xia, Nano Lett., 2008, 8, 2077-2081.

32 W. B. Smith, Tetrahedron, 2002, 58, 2091-2094.

33 A. Yan, X. H. Liu, G. Qiu, N. Zhang, R. R. Shi, R. Yi, M. Tang and R. C. Che, Solid State Commun., 2007, 144, 315-318.

34 X. W. Lou, L. A. Archer and Z. C. Yang, Adv. Mater., 2008, 20, 3987-4019.

35 S. W. Cao, Y. J. Zhu and J. Chang, New J. Chem., 2008, 32, 1526-1530.

36 N. E. Leadbeater, Microwave heating as a tool for sustainable chemistry, CRC Press, Boca Raton, 2012. 